\title{
Measurement of Density, Refractive Index and Conductance of Some Pyrimidine Derivatives in Solutions at 308.15 K.
}

\author{
Shipra Baluja*, Rahul Bhalodia and Ravi Gajera \\ Department of Chemistry, University of Saurashtra, India
}

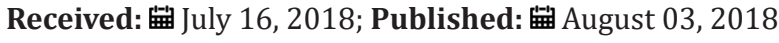

*Corresponding author: Shipra Baluja, Department of Chemistry, University of Saurashtra, Rajkot-360005, Gujarat, India

\begin{abstract}
Some new pyrimidine derivatives have been synthesized and their physicochemical parameters such as density, refractive index and conductance have been measured in $\mathrm{N}, \mathrm{N}$-dimethyl formamide and tetrahydrofuran at $308.15 \mathrm{~K}$. It is observed that solvent and structure of compounds have major effect on these studied properties which may be due to different type of interactions in different solvents for different compounds.

Keywords: Density; Refractive Index; Conductance; N, N-Dimethyl Formamide; Tetrahydrofuran
\end{abstract}

\section{Introduction}

Among many heterocyclic compounds, pyrimidine compounds are one of the most prominent structures which attract many chemists and pharmacists due to their therapeutic values [110]. The applications of these compounds in drug research have stimulated the invention of wide range of synthetic methods for their preparation and chemical transformations [11-15]. Further, many of these compounds are known to exist in deoxyribonucleic acid and ribonucleic acid which is one of the most essential constituents of all cells and thus of all living matter. As many of these compounds exist in various drugs [16-18], it would be useful to study their physical parameters which are prime factors for the study of these compounds in pharmaceutical field [19-21]. Thus, in the present work, physicochemical parameters such as density, refractive index and conductance of some newly synthesized pyrimidine derivatives such as dihydropyrimidine and dihydropyrimidinthiones have been measured in $\mathrm{N}, \mathrm{N}$-dimethyl formamide, dimethylsulphoxide and tetrahydrofuran at $308.15 \mathrm{~K}$.

\section{Experimental}

Some new dihydropyrimidine and dihydropyrimidinthiones compounds have been synthesized. The general structures and substitutions in different compounds are given in Figure 1.

\section{pysicochemical Studies}

The solvents DMF, DMSO and THF used for physicochemical studies were purified by fractionally distillation by the reported method [22]. For each compound, a series of solutions of different concentrations were prepared in these solvents. The choice of different solvents for dihydropyrimidine and dihydropyrimidinthiones is due to solubility problem. The density and refractive index of pure solvents and solutions were measured by using pycnometer and Abbe refractometer respectively at 308.15 K. The temperature was maintained by circulating water through jacket around the prisms of refractometer from an electronically controlled thermostatic water bath (NOVA NV-8550 E). The uncertainty of temperature was $\pm 0.1^{\circ} \mathrm{C}$. The conductance of each solution was measured by using Elico Conductivity Meter (Model No. CM 180) at $308.15 \mathrm{~K}$. The measured conductance was corrected by subtracting the conductance of pure solvent.

\section{Results and Discussion}

Table 1 shows the experimental values of densities and refractive index for all the studied solutions. Using experimental density of solution, density of each compound was calculated using the following relation:

$$
\frac{1}{\rho_{12}}=\frac{g_{1}}{\rho_{1}}+\frac{g_{2}}{\rho_{2}}
$$

where $\rho_{12}$ is the density of solution and $\rho_{1}$ and $\rho_{2}$ are the densities of solvent and solute respectively. $g_{1}$ and $g_{2}$ are the weight fractions of solvent and solute.

The evaluated densities of all the compounds are listed in (Table 2) along with the theoretical densities which were calculated using the following equation [23]: 
Table 1: The density $\left(\rho_{12}\right)$ and refractive index $(\mathrm{n})$ of synthesized compounds at $308.15 \mathrm{~K}$.

\begin{tabular}{|c|c|c|c|c|}
\hline $\begin{array}{c}\text { Conc. } \\
\text { M }\end{array}$ & $\begin{array}{c}\rho_{12} \\
\text { g. } \mathrm{cm}^{-3}\end{array}$ & $\mathbf{n}$ & $\begin{array}{c}\rho_{12} \\
\text { g. } \mathrm{cm}^{-3}\end{array}$ & $\mathbf{n}$ \\
\hline \multicolumn{5}{|c|}{ Dihydropyrimidinthiones } \\
\hline & \multicolumn{2}{|c|}{ DMF } & \multicolumn{2}{|c|}{ THF } \\
\hline \multicolumn{5}{|c|}{ SSN-1 } \\
\hline 0.00 & 0.9338 & 1.4239 & 0.8798 & 1.4016 \\
\hline 0.01 & 0.9413 & 1.4241 & 0.8832 & 1.4038 \\
\hline 0.02 & 0.9433 & 1.4249 & 0.8868 & 1.4043 \\
\hline 0.04 & 0.9470 & 1.4261 & 0.8898 & 1.4059 \\
\hline 0.06 & 0.9481 & 1.4275 & 0.8941 & 1.4076 \\
\hline 0.08 & 0.9523 & 1.4289 & 0.8972 & 1.4082 \\
\hline 0.10 & 0.9569 & 1.4313 & 0.9005 & 1.4097 \\
\hline \multicolumn{5}{|c|}{ SSN -2 } \\
\hline 0.01 & 0.9427 & 1.4242 & 0.8822 & 1.4032 \\
\hline 0.02 & 0.9447 & 1.4248 & 0.8843 & 1.4041 \\
\hline 0.04 & 0.9453 & 1.4259 & 0.8863 & 1.4057 \\
\hline 0.06 & 0.9466 & 1.4273 & 0.8891 & 1.4063 \\
\hline 0.08 & 0.9485 & 1.4291 & 0.8925 & 1.4076 \\
\hline 0.10 & 0.9494 & 1.4308 & 0.8962 & 1.4082 \\
\hline \multicolumn{5}{|c|}{ SSN -3 } \\
\hline 0.01 & 0.9409 & 1.4243 & 0.8840 & 1.4035 \\
\hline 0.02 & 0.9418 & 1.4248 & 0.8864 & 1.4053 \\
\hline 0.04 & 0.9462 & 1.4257 & 0.8886 & 1.4069 \\
\hline 0.06 & 0.9484 & 1.4268 & 0.8917 & 1.4083 \\
\hline 0.08 & 0.9495 & 1.4289 & 0.8941 & 1.4098 \\
\hline 0.10 & 0.9553 & 1.4292 & 0.8978 & 1.4111 \\
\hline \multicolumn{5}{|c|}{ SSN -4 } \\
\hline 0.01 & 0.9434 & 1.4247 & 0.8832 & 1.4031 \\
\hline 0.02 & 0.9457 & 1.4255 & 0.8861 & 1.4044 \\
\hline 0.04 & 0.9471 & 1.4265 & 0.8900 & 1.4069 \\
\hline 0.06 & 0.9485 & 1.4277 & 0.8948 & 1.4087 \\
\hline 0.08 & 0.9504 & 1.4286 & 0.8981 & 1.4093 \\
\hline 0.10 & 0.9527 & 1.4298 & 0.9002 & 1.4118 \\
\hline \multicolumn{5}{|c|}{ SSN -5 } \\
\hline 0.01 & 0.9421 & 1.4249 & 0.8845 & 1.4056 \\
\hline 0.02 & 0.9438 & 1.4256 & 0.8872 & 1.4061 \\
\hline 0.04 & 0.9468 & 1.4269 & 0.8898 & 1.4082 \\
\hline 0.06 & 0.9479 & 1.4289 & 0.8925 & 1.4092 \\
\hline 0.08 & 0.9494 & 1.4292 & 0.8960 & 1.4122 \\
\hline 0.10 & 0.9512 & 1.4311 & 0.8991 & 1.4138 \\
\hline \multicolumn{5}{|c|}{ SSN -6 } \\
\hline 0.00 & 0.9338 & 1.4239 & 0.8798 & 1.4016 \\
\hline 0.01 & 0.9431 & 1.4250 & 0.8812 & 1.4036 \\
\hline 0.02 & 0.9448 & 1.4258 & 0.8862 & 1.4052 \\
\hline 0.04 & 0.9454 & 1.4270 & 0.8904 & 1.4065 \\
\hline
\end{tabular}




\begin{tabular}{|c|c|c|c|c|}
\hline 0.06 & 0.9481 & 1.4282 & 0.8935 & 1.4079 \\
\hline 0.08 & 0.9521 & 1.4291 & 0.8962 & 1.4091 \\
\hline 0.10 & 0.9574 & 1.4305 & 0.8992 & 1.4108 \\
\hline \multicolumn{5}{|c|}{ SSN -7 } \\
\hline 0.01 & 0.9431 & 1.4253 & 0.8836 & 1.4034 \\
\hline 0.02 & 0.9454 & 1.4264 & 0.8865 & 1.4049 \\
\hline 0.04 & 0.9471 & 1.4276 & 0.8905 & 1.4059 \\
\hline 0.06 & 0.9489 & 1.4290 & 0.8954 & 1.4070 \\
\hline 0.08 & 0.9506 & 1.4308 & 0.8982 & 1.4082 \\
\hline 0.10 & 0.9514 & 1.4320 & 0.9022 & 1.4098 \\
\hline \multicolumn{5}{|c|}{ SSN -8 } \\
\hline 0.01 & 0.9413 & 1.4242 & 0.8871 & 1.4031 \\
\hline 0.02 & 0.9437 & 1.4247 & 0.8894 & 1.4046 \\
\hline 0.04 & 0.9450 & 1.4269 & 0.8924 & 1.4056 \\
\hline 0.06 & 0.9486 & 1.4289 & 0.8947 & 1.4079 \\
\hline 0.08 & 0.9533 & 1.4295 & 0.8981 & 1.4086 \\
\hline 0.10 & 0.9552 & 1.4312 & 0.9002 & 1.4112 \\
\hline \multicolumn{5}{|c|}{ SSN -9 } \\
\hline 0.01 & 0.9420 & 1.4243 & 0.8864 & 1.4034 \\
\hline 0.02 & 0.9456 & 1.4269 & 0.8892 & 1.4048 \\
\hline 0.04 & 0.9482 & 1.4272 & 0.8942 & 1.4058 \\
\hline 0.06 & 0.9512 & 1.4281 & 0.8974 & 1.4066 \\
\hline 0.08 & 0.9532 & 1.4309 & 0.9005 & 1.4079 \\
\hline 0.10 & 0.9563 & 1.4320 & 0.9044 & 1.4091 \\
\hline \multicolumn{5}{|c|}{ Dihydropyrimidine } \\
\hline & \multicolumn{2}{|c|}{ DMF } & \multicolumn{2}{|c|}{ DMSO } \\
\hline \multicolumn{5}{|c|}{ SNO-1 } \\
\hline 0.00 & 0.9338 & 1.4239 & 1.0959 & 1.4716 \\
\hline 0.01 & 0.9494 & 1.4261 & 1.0971 & 1.4719 \\
\hline 0.02 & 0.9506 & 1.4274 & 1.0982 & 1.4729 \\
\hline 0.04 & 0.9531 & 1.4291 & 1.0989 & 1.4733 \\
\hline 0.06 & 0.9555 & 1.4308 & 1.0998 & 1.4739 \\
\hline 0.08 & 0.9577 & 1.4321 & 1.1004 & 1.4745 \\
\hline 0.10 & 0.9584 & 1.4336 & 1.1012 & 1.4779 \\
\hline \multicolumn{5}{|c|}{ SNO -2 } \\
\hline 0.01 & 0.9485 & 1.4237 & 1.0971 & 1.4722 \\
\hline 0.02 & 0.9498 & 1.4248 & 1.0987 & 1.4729 \\
\hline 0.04 & 0.9516 & 1.4257 & 1.0992 & 1.4732 \\
\hline 0.06 & 0.9536 & 1.4275 & 1.1005 & 1.4739 \\
\hline 0.08 & 0.9554 & 1.4283 & 1.1017 & 1.4746 \\
\hline 0.10 & 0.9571 & 1.4308 & 1.1023 & 1.4759 \\
\hline \multicolumn{5}{|c|}{ SNO -3 } \\
\hline 0.01 & 0.9488 & 1.4223 & 1.0965 & 1.4728 \\
\hline 0.02 & 0.9490 & 1.4231 & 1.0973 & 1.4733 \\
\hline 0.04 & 0.9520 & 1.4235 & 1.1002 & 1.4741 \\
\hline 0.06 & 0.9527 & 1.4241 & 1.1009 & 1.4749 \\
\hline
\end{tabular}




\begin{tabular}{|c|c|c|c|c|}
\hline 0.08 & 0.9543 & 1.4249 & 1.1010 & 1.4762 \\
\hline 0.10 & 0.9565 & 1.4265 & 1.1026 & 1.4779 \\
\hline \multicolumn{5}{|c|}{ SNO -4 } \\
\hline 0.01 & 0.9470 & 1.4219 & 1.0974 & 1.4728 \\
\hline 0.02 & 0.9474 & 1.4226 & 1.0982 & 1.4735 \\
\hline 0.04 & 0.9478 & 1.4231 & 1.0992 & 1.4748 \\
\hline 0.06 & 0.9484 & 1.4238 & 1.0996 & 1.4762 \\
\hline 0.08 & 0.9489 & 1.4249 & 1.1010 & 1.4778 \\
\hline 0.10 & 0.9505 & 1.4255 & 1.1027 & 1.4783 \\
\hline \multicolumn{5}{|c|}{ SNO -5 } \\
\hline 0.01 & 0.9494 & 1.4225 & 1.0969 & 1.4719 \\
\hline 0.02 & 0.9517 & 1.4234 & 1.0985 & 1.4723 \\
\hline 0.04 & 0.9530 & 1.4243 & 1.0996 & 1.4729 \\
\hline 0.06 & 0.9555 & 1.4253 & 1.1003 & 1.4741 \\
\hline 0.08 & 0.9577 & 1.4258 & 1.1017 & 1.4749 \\
\hline 0.10 & 0.9597 & 1.4283 & 1.1036 & 1.4756 \\
\hline \multicolumn{5}{|c|}{ SNO -6 } \\
\hline 0.00 & 0.9453 & 1.4218 & 1.0971 & 1.4722 \\
\hline 0.01 & 0.9487 & 1.4219 & 1.0987 & 1.4729 \\
\hline 0.02 & 0.9505 & 1.4227 & 1.0992 & 1.4732 \\
\hline 0.04 & 0.9518 & 1.4233 & 1.1005 & 1.4739 \\
\hline 0.06 & 0.9547 & 1.4249 & 1.1017 & 1.4746 \\
\hline 0.08 & 0.9554 & 1.4259 & 1.1023 & 1.4759 \\
\hline 0.10 & 0.9566 & 1.4273 & 1.0971 & 1.4722 \\
\hline \multicolumn{5}{|c|}{ SNO -7 } \\
\hline 0.01 & 0.9485 & 1.4253 & 0.8836 & 1.4034 \\
\hline 0.02 & 0.9500 & 1.4264 & 0.8865 & 1.4049 \\
\hline 0.04 & 0.9515 & 1.4276 & 0.8905 & 1.4059 \\
\hline 0.06 & 0.9528 & 1.4290 & 0.8954 & 1.4070 \\
\hline 0.08 & 0.9535 & 1.4308 & 0.8982 & 1.4082 \\
\hline 0.10 & 0.9546 & 1.4320 & 0.9022 & 1.4098 \\
\hline \multicolumn{5}{|c|}{ SNO -8 } \\
\hline 0.01 & 0.9505 & 1.4219 & 0.8871 & 1.4031 \\
\hline 0.02 & 0.9523 & 1.4224 & 0.8894 & 1.4046 \\
\hline 0.04 & 0.9558 & 1.4230 & 0.8924 & 1.4056 \\
\hline 0.06 & 0.9581 & 1.4236 & 0.8947 & 1.4079 \\
\hline 0.08 & 0.9587 & 1.4241 & 0.8981 & 1.4086 \\
\hline 0.10 & 0.9595 & 1.4249 & 0.9002 & 1.4112 \\
\hline \multicolumn{5}{|c|}{ SNO -9 } \\
\hline 0.01 & 0.9508 & 1.4224 & 0.8864 & 1.4034 \\
\hline 0.02 & 0.9524 & 1.4232 & 0.8892 & 1.4048 \\
\hline 0.04 & 0.9558 & 1.4239 & 0.8942 & 1.4058 \\
\hline 0.06 & 0.9576 & 1.4252 & 0.8974 & 1.4066 \\
\hline 0.08 & 0.9605 & 1.4268 & 0.9005 & 1.4079 \\
\hline 0.10 & 0.9617 & 1.4281 & 0.9044 & 1.4091 \\
\hline
\end{tabular}


Table 2: Experimental and theoretical densities of compounds at $308.15 \mathrm{~K}$.

\begin{tabular}{|c|c|c|c|}
\hline \multicolumn{4}{|c|}{ Dihydropyrimidinthiones } \\
\hline \multirow{2}{*}{ Compound Code } & \multicolumn{2}{|c|}{ Experimental Density (g.cm ${ }^{-3}$ ) } & \multirow{2}{*}{ Theoretical Density $\left(\mathrm{g} \cdot \mathrm{cm}^{-3}\right)$} \\
\hline & DMF & THF & \\
\hline SSN -1 & 1.7358 & 1.6886 & 1.3512 \\
\hline SSN -2 & 1.3335 & 1.4658 & 1.3682 \\
\hline SSN -3 & 1.6717 & 1.4804 & 1.3782 \\
\hline SSN -4 & 1.4420 & 1.7781 & 1.4649 \\
\hline SSN -5 & 1.4205 & 1.5618 & 1.4731 \\
\hline SSN -6 & 1.6642 & 1.6393 & 1.4689 \\
\hline SSN -7 & 1.3714 & 1.7655 & 1.4689 \\
\hline SSN -8 & 1.6753 & 1.5265 & 1.4714 \\
\hline SSN -9 & 1.6554 & 1.8272 & 1.5120 \\
\hline \multicolumn{4}{|c|}{ Dihydropyrimidine } \\
\hline & DMF & DMSO & \\
\hline SNO-1 & 1.3746 & 1.2347 & 1.0526 \\
\hline SNO -2 & 1.3226 & 1.3201 & 1.0749 \\
\hline SNO -3 & 1.2560 & 1.3168 & 1.1127 \\
\hline SNO -4 & 1.0622 & 1.3006 & 1.0749 \\
\hline SNO -5 & 1.1847 & 1.3355 & 1.0526 \\
\hline SNO -6 & 1.2962 & 1.3029 & 1.1319 \\
\hline SNO -7 & 1.2596 & 1.2999 & 1.1127 \\
\hline SNO -8 & 1.3454 & 1.3186 & 1.1588 \\
\hline SNO -9 & 1.3263 & 1.2204 & 1.1695 \\
\hline
\end{tabular}

$$
\rho=K M / N_{A} \sum \Delta V_{i}
$$

where $\rho$ is the density of the compound, $\mathrm{K}$ is packing fraction (0.599), $M$ is the molecular weight of the compound, $N_{A}$ is the Avogadro's number and $\Delta V_{i}$ is the volume increment of the atoms and atomic groups present in the compound.

Comparison of densities values showed that theoretical density values are different from those evaluated from experimental data. Further, for the same compound, density in the two solvents is different. This suggests that solvent plays an important role. In solutions, compounds interact differently depending upon their substitution, structure and nature of solvent. These molecular interactions affect volume which causes change in density.

Further, the molar refraction of a pure liquid (MRD)1 were calculated by the following equation:

$$
(M R D)_{1}=\left[\frac{n^{2}-1}{n^{2}+1}\right] \frac{M}{\rho}
$$

where $n, M$ and $\rho$ are refractive index, molecular weight and density pure liquid respectively.

For solutions, the following equation was used to determining molar refraction:

$$
(M R D)_{12}=\left[\frac{n_{12}^{2}-1}{n_{12}^{2}+1}\right]\left[\frac{X_{1} M_{1}+X_{2} M_{2}}{\rho_{12}}\right]
$$

where $\mathrm{n}_{12}$ and $\rho_{12}$ are refractive index and density of solution respectively. $X_{1}$ and $X_{2}$ are the mole fractions and $M_{1}$ and $M_{2}$ are the molecular weight of the solvent and solute respectively.

From the values of the molar refraction of solution and pure solvent, molar refraction of solid compounds was determined by following equation:

$$
(M R D)_{12}=X_{1}(M R D)_{1}+X_{2}(M R D)_{2}
$$

From the density and molar refraction data, the refractive indexes of all the compounds were calculated from equation (5). The molar refraction (MRD) $)_{2}$ and refractive index of all the compounds are reported in Table 3 for $0.1 \mathrm{M}$ solution.

It is evident from Table 3 that both (MRD) ${ }_{2}$ and refractive index of compounds are different in each solvent. This again proves that in different solvents, intermolecular interactions are different, which affect these parameters. In some solvents, there may be interaction between solute and solvent molecules where as in others breakage of bonds may take place. As refractive index and molar refraction depends not only upon atomic refraction but also upon single, double or triple bonds, these parameters are affected by the type of interactions taking place in solution. Further, bond polarity also causes change in molar refraction. Thus, type of solvent affects the refractive index and molar refraction of a solute. The measured conductance $(\mathrm{k})$ of each solution after correction is listed in Table 4. It is observed that for all the studied compounds, conductance increases with concentration in all the solvents. For 
dihydropyrimidinthiones compounds, conductance is lower in THF than that of DMF which may be due to greater electro relaxation effect owing to the higher permittivity of THF, which contributes interionic repulsions to a larger extent [24]. For dihydropyrimidine, conductivity is less in DMSO than that in DMF.

Table 3: Molar refraction (MRD) ${ }_{2}$ and refractive index (n) of 0.1 $\mathrm{M}$ solutions of compounds at $308.15 \mathrm{~K}$.

\begin{tabular}{|c|c|c|c|c|}
\hline \multirow{2}{*}{ Compounds } & \multicolumn{4}{|c|}{ Dihydropyrimidinthiones } \\
\cline { 2 - 5 } & \multicolumn{2}{|c|}{ DMF } & \multicolumn{2}{c|}{ THF } \\
\cline { 2 - 5 } & (MRD $_{2}$ ) & n & (MRD $_{2}$ ) & n \\
\hline SSN - & 91.0744 & 1.6724 & 102.4883 & 1.7566 \\
\hline SSN -2 & 100.7345 & 1.6012 & 98.1486 & 1.6551 \\
\hline SSN -3 & 80.0690 & 1.5734 & 113.1660 & 1.7628 \\
\hline SSN -4 & 91.4046 & 1.5561 & 111.3178 & 1.9494 \\
\hline SSN -5 & 101.7629 & 1.6267 & 124.5989 & 1.9328 \\
\hline
\end{tabular}

\begin{tabular}{|c|c|c|c|c|}
\hline SSN -6 & 86.6130 & 1.5919 & 113.1603 & 1.8205 \\
\hline SSN -7 & 113.8506 & 1.6333 & 102.4138 & 1.7656 \\
\hline SSN -8 & 96.2845 & 1.6817 & 112.5131 & 1.7397 \\
\hline SSN -9 & 100.4741 & 1.6862 & 92.4797 & 1.7004 \\
\hline \multicolumn{5}{|c|}{ Dihydropyrimidine } \\
\hline \multirow{4}{*}{ DMF } & DMSO \\
\cline { 2 - 5 } & (MRD $_{\mathbf{2}}$ ) & $\mathbf{n}$ & $\mathbf{~ ( M R D ~}_{\mathbf{2}}$ ) & $\mathbf{n}$ \\
\hline SNO-1 & 133.61 & 1.4336 & 129.88 & 1.4779 \\
\hline SNO -2 & 118.08 & 1.4308 & 102.81 & 1.4759 \\
\hline SNO -3 & 102.37 & 1.4265 & 117.35 & 1.4779 \\
\hline SNO -4 & 107.93 & 1.4255 & 114.02 & 1.4783 \\
\hline SNO -5 & 181.09 & 1.4283 & 102.03 & 1.4756 \\
\hline SNO -6 & 106.33 & 1.4273 & 117.85 & 1.4781 \\
\hline SNO -7 & 105.32 & 1.4269 & 112.75 & 1.4779 \\
\hline SNO -8 & 95.20 & 1.4249 & 124.21 & 1.4784 \\
\hline SNO -9 & 106.18 & 1.4281 & 131.64 & 1.4787 \\
\hline
\end{tabular}

Table 4: Molar refraction (MRD) ${ }_{2}$ and refractive index (n) of $0.1 \mathrm{M}$ solutions of compounds at $308.15 \mathrm{~K}$.

\begin{tabular}{|c|c|c|c|c|c|c|c|c|c|c|}
\hline $\begin{array}{c}\text { Conc. } \\
\text { M }\end{array}$ & \multicolumn{10}{|c|}{ k.10 ${ }^{5} \mathrm{mho}$} \\
\hline \multicolumn{11}{|c|}{ Dihydropyrimidinthiones } \\
\hline \multicolumn{11}{|c|}{ DMF } \\
\hline & SSN -1 & SSN -2 & SSN -3 & SSN -4 & SSN -5 & SSN -6 & SSN -7 & SSN -8 & SSN -9 & SSN-10 \\
\hline 0.000 & 0.200 & 0.200 & 0.200 & 0.200 & 0.200 & 0.200 & 0.200 & 0.200 & 0.200 & 0.200 \\
\hline 0.001 & 0.325 & 0.420 & 0.380 & 0.715 & 1.200 & 0.900 & 0.568 & 0.817 & 0.418 & 0.665 \\
\hline 0.002 & 0.391 & 0.624 & 0.480 & 0.910 & 2.040 & 1.420 & 0.614 & 1.020 & 0.577 & 1.000 \\
\hline 0.004 & 0.550 & 0.997 & 0.605 & 1.320 & 3.720 & 1.780 & 0.728 & 1.080 & 0.752 & 1.610 \\
\hline 0.006 & 0.656 & 1.340 & 0.610 & 1.580 & 5.000 & 1.830 & 0.880 & 1.380 & 0.830 & 2.120 \\
\hline 0.008 & 0.790 & 1.700 & 0.612 & 1.820 & 5.410 & 1.900 & 0.980 & 1.410 & 0.980 & 2.570 \\
\hline 0.010 & 0.900 & 1.910 & 0.630 & 2.090 & 5.700 & 2.050 & 1.090 & 1.620 & 1.000 & 2.980 \\
\hline 0.020 & 1.500 & 3.350 & 1.030 & 3.500 & 8.800 & 3.190 & 1.630 & 2.500 & 1.500 & 3.530 \\
\hline 0.040 & 2.500 & 5.900 & 1.740 & 5.900 & 14.800 & 4.800 & 2.240 & 4.000 & 2.500 & 4.970 \\
\hline 0.060 & 3.500 & 8.450 & 2.410 & 6.300 & 19.300 & 5.620 & 3.000 & 5.000 & 3.400 & 6.390 \\
\hline 0.080 & 4.300 & 10.800 & 2.820 & 6.800 & 23.500 & 6.010 & 3.130 & 5.500 & 4.500 & 7.680 \\
\hline 0.100 & 4.900 & 12.800 & 3.300 & 7.200 & 24.000 & 6.600 & 3.440 & 6.250 & 5.600 & 8.270 \\
\hline \multicolumn{11}{|c|}{ THF } \\
\hline 0.000 & 0.024 & 0.024 & 0.024 & 0.024 & 0.024 & 0.024 & 0.024 & 0.024 & 0.024 & 0.024 \\
\hline 0.001 & 0.098 & 0.101 & 0.185 & 0.169 & 0.132 & 0.187 & 0.127 & 0.367 & 0.282 & 0.287 \\
\hline 0.002 & 0.101 & 0.107 & 0.318 & 0.181 & 0.224 & 0.292 & 0.144 & 0.594 & 0.454 & 0.450 \\
\hline 0.004 & 0.146 & 0.133 & 0.425 & 0.209 & 0.385 & 0.380 & 0.168 & 0.972 & 0.729 & 0.684 \\
\hline 0.006 & 0.177 & 0.173 & 0.498 & 0.285 & 0.507 & 0.504 & 0.188 & 1.240 & 0.828 & 0.853 \\
\hline 0.008 & 0.192 & 0.195 & 0.535 & 0.341 & 0.551 & 0.595 & 0.210 & 1.540 & 0.945 & 0.972 \\
\hline 0.010 & 0.207 & 0.200 & 0.580 & 0.390 & 0.570 & 0.660 & 0.235 & 1.670 & 1.040 & 1.010 \\
\hline 0.020 & 0.377 & 0.320 & 1.030 & 0.620 & 0.880 & 1.120 & 0.372 & 2.650 & 1.520 & 1.720 \\
\hline 0.040 & 0.701 & 0.590 & 1.740 & 1.010 & 1.480 & 1.720 & 0.561 & 3.940 & 2.690 & 2.220 \\
\hline 0.060 & 0.886 & 0.860 & 2.410 & 1.260 & 1.930 & 2.270 & 0.725 & 4.210 & 3.420 & 2.860 \\
\hline 0.080 & 0.955 & 1.080 & 2.840 & 1.590 & 2.350 & 2.730 & 0.878 & 4.970 & 4.280 & 3.440 \\
\hline
\end{tabular}




\begin{tabular}{|c|c|c|c|c|c|c|c|c|c|c|}
\hline 0.100 & 1.180 & 1.280 & 3.340 & 1.900 & 2.400 & 2.990 & 1.000 & 5.550 & 5.070 & 3.510 \\
\hline \multicolumn{11}{|c|}{ Dihydropyrimidine } \\
\hline \multicolumn{11}{|c|}{ DMF } \\
\hline & SNO -1 & SNO - 2 & SNO -3 & SNO -4 & SNO - 5 & SNO -6 & SNO -7 & SNO -8 & SNO -9 & SNO-10 \\
\hline 0.000 & 0.200 & 0.200 & 0.200 & 0.200 & 0.200 & 0.200 & 0.200 & 0.200 & 0.200 & 0.200 \\
\hline 0.001 & 0.390 & 0.322 & 0.400 & 0.422 & 0.300 & 0.310 & 0.422 & 0.320 & 0.261 & 0.550 \\
\hline 0.002 & 0.461 & 0.390 & 0.530 & 0.640 & 0.354 & 0.415 & 0.591 & 0.440 & 0.304 & 0.781 \\
\hline 0.004 & 0.672 & 0.532 & 0.847 & 0.993 & 0.494 & 0.552 & 0.982 & 0.622 & 0.377 & 1.142 \\
\hline 0.006 & 0.866 & 0.686 & 1.200 & 1.410 & 0.592 & 0.774 & 1.342 & 0.792 & 0.446 & 1.480 \\
\hline 0.008 & 0.993 & 0.863 & 1.491 & 1.820 & 0.690 & 0.983 & 1.751 & 0.984 & 0.521 & 1.843 \\
\hline 0.010 & 1.180 & 1.075 & 1.844 & 2.190 & 0.811 & 1.231 & 2.090 & 1.131 & 0.617 & 2.150 \\
\hline 0.020 & 2.140 & 1.674 & 3.420 & 5.700 & 1.151 & 2.090 & 3.630 & 2.160 & 0.833 & 3.210 \\
\hline 0.040 & 3.830 & 3.060 & 5.560 & 10.220 & 1.925 & 3.510 & 6.240 & 3.600 & 1.121 & 4.220 \\
\hline 0.060 & 5.190 & 4.100 & 7.230 & 14.800 & 2.360 & 4.620 & 9.090 & 4.740 & 1.324 & 5.960 \\
\hline 0.080 & 6.180 & 4.950 & 7.820 & 18.800 & 2.750 & 5.720 & 11.130 & 4.980 & 1.567 & 7.310 \\
\hline 0.100 & 7.030 & 5.700 & 8.520 & 22.600 & 3.200 & 6.410 & 13.440 & 5.300 & 1.775 & 9.22 \\
\hline \multicolumn{11}{|c|}{ DMSO } \\
\hline 0.000 & 0.016 & 0.016 & 0.016 & 0.016 & 0.016 & 0.016 & 0.016 & 0.016 & 0.016 & 0.016 \\
\hline 0.001 & 0.134 & 0.124 & 0.191 & 0.117 & 0.291 & 0.027 & 0.023 & 0.022 & 0.030 & 0.023 \\
\hline 0.002 & 0.237 & 0.177 & 0.286 & 0.197 & 0.524 & 0.038 & 0.028 & 0.029 & 0.045 & 0.027 \\
\hline 0.004 & 0.425 & 0.288 & 0.497 & 0.382 & 0.980 & 0.060 & 0.036 & 0.039 & 0.084 & 0.039 \\
\hline 0.006 & 0.652 & 0.375 & 0.678 & 0.548 & 1.350 & 0.082 & 0.045 & 0.050 & 0.121 & 0.051 \\
\hline 0.008 & 0.871 & 0.473 & 0.870 & 0.760 & 1.670 & 0.104 & 0.053 & 0.062 & 0.154 & 0.061 \\
\hline 0.010 & 1.010 & 0.520 & 1.070 & 1.020 & 2.040 & 0.124 & 0.159 & 0.078 & 0.214 & 0.110 \\
\hline 0.020 & 1.560 & 0.770 & 1.750 & 1.680 & 3.570 & 0.356 & 0.243 & 0.137 & 0.282 & 0.192 \\
\hline 0.040 & 2.700 & 1.320 & 3.070 & 3.020 & 3.780 & 0.567 & 0.385 & 0.248 & 0.371 & 0.301 \\
\hline 0.060 & 3.770 & 1.780 & 4.080 & 4.160 & 4.070 & 0.785 & 0.554 & 0.361 & 0.497 & 0.438 \\
\hline 0.080 & 5.370 & 2.260 & 5.010 & 5.340 & 4.530 & 0.883 & 0.718 & 0.451 & 0.620 & 0.562 \\
\hline 0.100 & 6.330 & 2.890 & 5.910 & 6.810 & 4.680 & 0.996 & 0.910 & 0.522 & 0.801 & 0.756 \\
\hline
\end{tabular}

From corrected conductance, specific conductance $(\kappa)$ and equivalent conductance $\left(\lambda_{c}\right)$ are calculated using the following equations:

$$
\begin{gathered}
\kappa=k \theta \\
\lambda_{c}=1000 \frac{\kappa}{C}
\end{gathered}
$$

where $\theta$ is the cell constant $\left(0.96 \mathrm{~cm}^{-1}\right)$ and $\mathrm{c}$ is the concentration (g. equi. /lit.) of solution.

Table 5: The limiting equivalent conductance $(\lambda 0)$ of some synthesized compounds at $308.15 \mathrm{~K}$.

\begin{tabular}{|c|c|c|}
\hline Compound code & \multicolumn{2}{|c|}{$\boldsymbol{\lambda}_{\mathbf{0}}$ mho.cm $^{\mathbf{2}}$. equi. ${ }^{-1}$} \\
\hline \multicolumn{3}{|c|}{ Dihydropyrimidinthiones } \\
\hline & DMF & THF \\
\hline SSN -1 & 2.8 & 1.5 \\
\hline SSN -2 & 5.2 & 1.68 \\
\hline SSN -3 & 3.6 & 2.3 \\
\hline SSN -4 & - & 2.5 \\
\hline SSN -5 & 12.7 & 1.5 \\
\hline
\end{tabular}

\begin{tabular}{|c|c|c|}
\hline SSN -6 & 8.5 & 2.29 \\
\hline SSN -7 & 4.7 & 2.52 \\
\hline SSN -8 & - & - \\
\hline SSN -9 & 3.1 & 3.5 \\
\hline SSN-10 & 6 & - \\
\hline \multicolumn{2}{|c|}{ Dihydropyrimidine } \\
\hline SNO-1 & - & DMF \\
\hline SNO -2 & 1.51 & 1.2 \\
\hline SNO -3 & - & - \\
\hline SNO -4 & 2.4 & - \\
\hline SNO -5 & - & 1.1 \\
\hline SNO -6 & - & 3 \\
\hline SNO -7 & 1.26 & 0.5 \\
\hline SNO -8 & 2.45 & 0.2 \\
\hline SNO -9 & 1.46 & 0.14 \\
\hline SNO-10 & 0.92 & 0.12 \\
\hline
\end{tabular}

Cite this article: Shipra B, Rahul B, Ravi G. Measurement of Density, Refractive Index and Conductance of Some Pyrimidine Derivatives in 
The equivalent conductance $\left(\lambda_{\mathrm{c}}\right)$ is plotted against $\sqrt{\mathrm{C}}$ for all compounds as shown in (Figures 1 \& 2). For all the dihydropyrimidinthiones and dihydropyrimidine, the equivalent conductance increases with concentration in both the solvents. It is obvious from figures that some of the compounds behave as strong electrolytes whereas others exhibited weak electrolytic behavior. The behavior is different in different solvents. For weak electrolytes, it is difficult to determine $\lambda_{0}$. However, for some of the compounds in the studied solutions of compounds, $\lambda_{0}$ values are evaluated approximately by extrapolation method and are reported in Table 5.

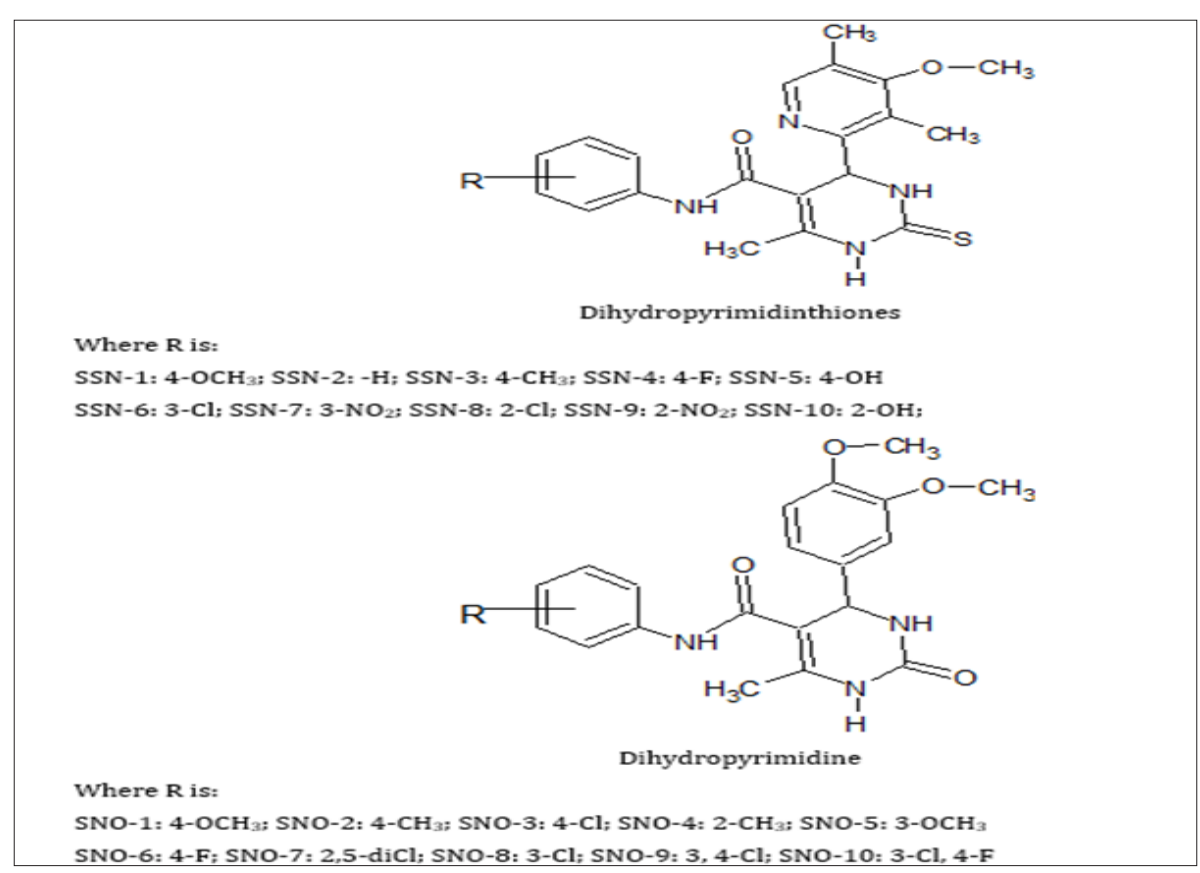

Figure 1: General structure of synthesized different pyrimidine derivatives.

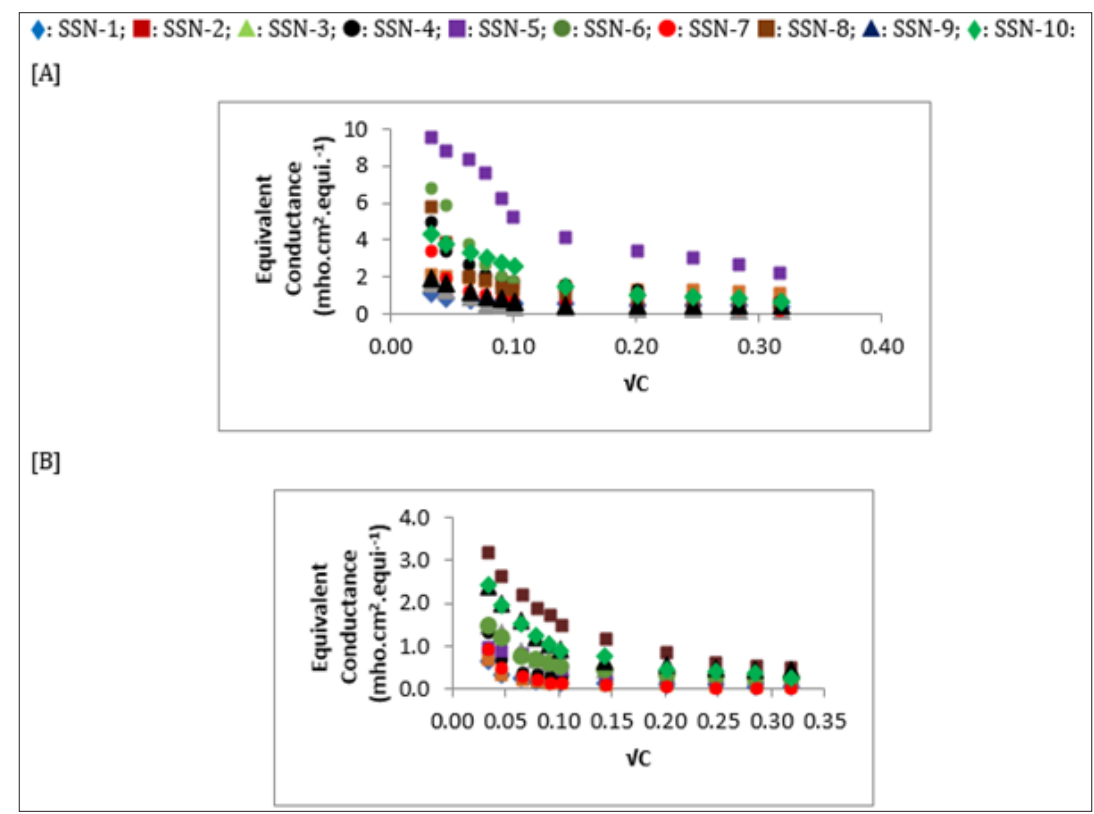

Figure 2: The variation of equivalent conductance with $₫ \mathrm{C}$ for Dihydro pyrimidinones in $[\mathrm{A}] \mathrm{DMF}$ and [B] DMSO at $308.15 \mathrm{~K}$.

\section{Conclusion}

It is observed that physicochemical parameters of compounds in solution depends not only on the structure and substitution of the compound but also on the nature of solvent in which it is dissolved.
The molecular interactions occurring in the solution affect volume which in turn causes a small change in density and refractive index. Depending upon the nature of solvent, the conductance i.e., electrolytic behavior of compounds also changes. 


\section{References}

1. KN Mohana, BNP Kumar, L Mallesha (2013) Synthesis and biological activity of some pyrimidine derivatives. Drug Inven Today 5(3): 216222.

2. S Gorle, S Maddila, S Chokkakula, $\mathrm{P}$ Lavanya, $\mathrm{M}$ Singh, et al (2016) Synthesis, Biological Activity of Pyrimidine Linked with Morpholinophenyl Derivatives. J Heterocyc Chem 53: 1852-1858.

3. S El Bahaie, AM Kadry, MG Assy, YA Ibrahim (1988) Synthesis and biological activity of some new pyrimidine and thienopyrimidine derivatives. Pharmazie 43(8): 537-538.

4. A Kumar, S Sinha, PM Chauhan (2012) Synthesis of novel antimycobacterial combinatorial libraries of structurally diverse substituted pyrimidines by three-component solid phase reactions. Bioorg Med Chem Lett 12(4): 667-669.

5. PG Baraldi, MG Pavani, M Nunez (2002) Antimicrobial and antitumor activity of $\mathrm{N}$-heteroimine-1,2,3-dithiazoles and their transformation in triazolo- imidazo- and pyrazolo pyrimidines. Bioorg Med Chem 10(2): 449-456.

6. A Gangjee, A Vidwans, E Elzein, JJ McGuire, SF Queener, et al. (2001) Synthesis, antifolate and antitumor activities of classical and nonclassical 2-amino-4-oxo-5- substituted-pyrrolo [2,3-d] pyrimidines, J Med Chem 44(12): 1993-2003.

7. AN Chiang, JC Valderramos, R Balachandran, RJ Chovatiya, BP Mead (2009) Select pyrimidinones inhibit the propagation of the malarial parasite, Plasmodium falciparum. Bioorg Med Chem 17(4): 1527-1533.

8. HA Stefani, CB Oliveira, RB Almeida, CMP Pereira, RC Braga (2006) Dihydropyridine- $(2 \mathrm{H})$-ones obtained by ultrasound irradiation: A new class of potential antioxidant agents. Eur J Med Chem 41(4): 513-518.

9. T Gregorić, M Sedić, P Grbčić, AT Paravić, SK Pavelić, et al. (2017) Novel pyrimidine-2,4-dione-1,2,3-triazole and furo[2,3-d] pyrimidine-2one-1,2,3-triazole hybrids as potential anti-cancer agents: Synthesis, computational and X-ray analysis and biological evaluation. Eur J Med Chem 125: 1247-1267.

10. FM Awadalla, GA Piazz, BD Gar, AB Keeto, JC Canzoner (2013) Synthesis of some dihydropyrimidine-based compounds bearing pyrazoline moiety and evaluation of their antiproliferative activity. Eur J Med Chem 70: 273-279.

11. M Movassaghi, MD Hill (2006) Single-Step Synthesis of Pyrimidine Derivatives. J Am Chem Soc 128(44): 14254-14255.

12. M Mahfoudh, R Abderrahim, E Leclerc, JM Campagne (2017) Recent Approaches to the Synthesis of Pyrimidine Derivatives. Eur J Org Chem 2017(20): 2856-2865.
13. JB Shi, WJ Tang, X Baoqi, R Li, XH Liu (2015) Novel pyrazole-5carboxamide and pyrazole-pyrimidine derivatives: Synthesis and anticancer activity. Eur J Med Chem 90: 889-896.

14. SN Maddila, S Maddila, WE Van Zyl, SB Jonnalagadda (2015) Mn doped $\mathrm{ZrO}_{2}$ as a green, efficient and reusable heterogeneous catalyst for the multicomponent synthesis of pyrano [2,3-d]-pyrimidine derivatives. RSC Adv 5(47): 37360-37366.

15. G Cui, J Jin, H Chen, R Cao, X Chen, et al. (2018) Synthesis and biological evaluation of pyrimidine derivatives as novel human Pin1 inhibitors. Bioorg Med Chem 26(8): 2186-2197.

16. Q Tu, M Eckelman, J Zimmerman (2017) Meta-analysis and Harmonization of Life Cycle Assessment Studies for Algae Biofuels. Environ Sci Technol 51(17): 9419-9432.

17. TH Chen, CF Chang, SC Yu, JC Wang, CH Chen, et al. (2009) Dipyridamole inhibits cobalt chloride-induced osteopontin expression in NRK52E cells. Eur J Pharmacol 613(1-3): 10-18.

18. CM Marson (2017) Saturated Heterocycles with Applications in Medicinal Chemistry. Adv Heterocyc Chem 121: 13-33.

19. L Zhang, M Xin, H Shen, J Wen, F Tang, et al. (2014) Five - membered hetero aromatic ring fused-pyrimidine derivatives: design, synthesis, and hedgehog signaling pathway inhibition study. Bioorg Med Chem Lett 24(15): 3486-3492.

20. V Kanagarajan, J Thanusu, M Gopalakrishnan (2009) A green chemical approach towards the 'one-pot' synthesis, spectral characterization and in vitro antibacterial and antifungal activities of morpholino pyrimidines. J Korean Chem Soc 53(6): 731-741.

21. M Ashid, P Yogi, D Katariya, P Agarwal, A Joshi (2016) Pyrimidine: medicinal and biological significance a review. World J Pharm Pharmaceutical Sci 5(9): 990-1009.

22. JA Riddick, WB Bunger, T Sakano (1986) Organic Solvents-Physical Properties and methods of purification. Techniques of Chemistry II, A Wiley Interscience Publication, John Wiley, New York, USA.

23. GL Slonimskii, AA Askadshii, AI Kitaigorodskii (1970) Vysokomol Soyed 12: 494-498.

24. M Singh, BB Prasad (1996) Electrolytic conductivity of the N-chloraniland $\mathrm{N}$-xylylene- based polyelectrolytes in dimethylformamide and dimethyl sulfoxide. J Chem Eng Data 41(3): 409-413.

\section{ISSN: 2574-1241}

DOI: 10.26717/BJSTR.2018.07.001532

Shipra Baluja. Biomed J Sci \& Tech Res

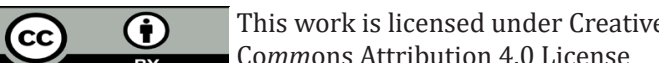

Submission Link: https://biomedres.us/submit-manuscript.php

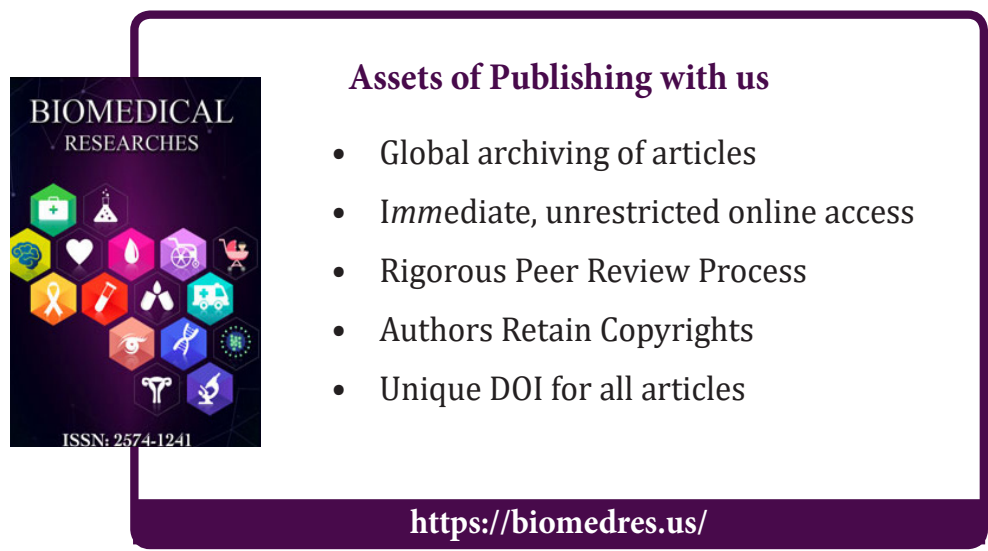

